\title{
Une stratégie pour la réduction de la mortalité maternelle et néonatale
}

Population Council

Follow this and additional works at: https://knowledgecommons.popcouncil.org/departments_sbsr-rh

Part of the Health Policy Commons, International Public Health Commons, Maternal and Child Health Commons, Public Health Education and Promotion Commons, and the Women's Health Commons How does access to this work benefit you? Let us know!

\section{Recommended Citation}

Population Council. 2010. "Une stratégie pour la réduction de la mortalité maternelle et néonatale," brief. Dakar: Population Council. 


\section{Sénégal}

SMNI/PF/PALUDISME

\section{La Situation 1}

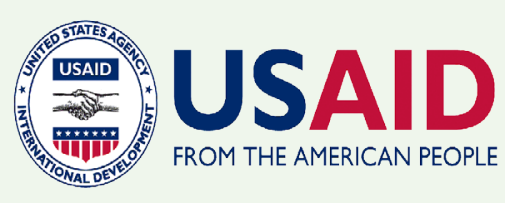

\section{Une stratégie pour la réduction de} la mortalité maternelle et néonatale

Dans le cadre des stratégies visant à réduire la mortalité maternelle et néonatale, l'introduction d'un paquet minimum intégré de soins pour la mère et le nouveau-né incluant la Gestion Active de la Troisième Phase de l'Accouchement (GATPA) et l'administration de Soins Essentiels au Nouveau-Né (SENN), constituent des approches simples et peu coûteuses dans des pays pauvres.

\section{Contexte}

Le taux de mortalité maternelle est de 401 décès pour 100000 naissances vivantes (EDS/Sénégal, 2005). La période du postpartum immédiat constitue une période critique pour la survie de la mère et du nouveau-né avec $60 \%$ des décès maternels et environ $75 \%$ des décès de nouveau-nés survenant dans la première semaine de l'accouchement (Lancet, 2006). Dans le cadre des stratégies visant à réduire la mortalité maternelle et néonatale, l'introduction d'un paquet minimum intégré de soins pour la mère et le nouveau-né incluant la Gestion Active de la Troisième Phase de l'Accouchement (GATPA) et l'administration de Soins Essentiels au Nouveau-Né (SENN), constituent des approches simples et peu coûteuses dans des pays pauvres.

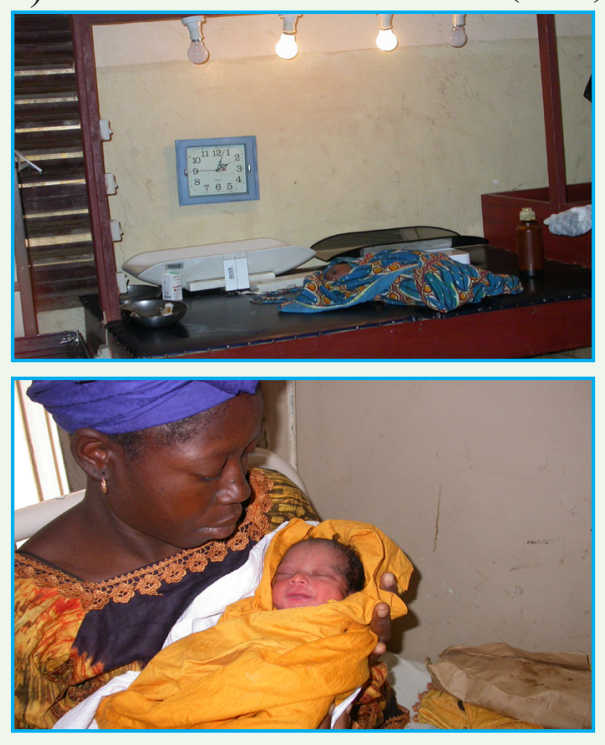

et les SENN; 2) évaluer les connaissances et les compétences des prestataires formés dans le domaine de la GATPA et des SENN ;3) identifier les problèmes rencontrés dans l'application de la GATPA et des soins essentiels aux nouveau-nés et 4) formuler des recommandations. Cette étude a touché 170 prestataires et 98 points de prestations de services (PPS) dans les 26 districts sanitaires des 5 régions ciblées dont les prestataires ont eu à bénéficier d'une formation sur la GATPA et les SENN. La capacité fonctionnelle des structures à offrir les services de GATPA et les SENN ainsi que la performance des prestataires ont été mesurées selon un système de score attribué par rapport à un niveau acceptable de conformité fixé à $100 \%$ ou à $85 \%$ pour les connaissances et compétences.
Le Ministère de la Santé et de la Prévention (MSP), à travers la Division de la Santé de la Reproduction (DSR), a initié, avec l'appui d'IntraHealth International, un important programme de renforcement des capacités des prestataires sur la GATPA intégrant les SENN au niveau des régions de Louga, Thiès, Kaolack, Kolda et Ziguinchor où le paquet intégré de $\mathrm{SMNI} / \mathrm{PF} /$ Paludisme a été mis en place.

Une étude a été menée avec pour objectifs de : 1) évaluer la capacité fonctionnelle des structures de santé ciblées à offrir la GATPA

\section{Résultats}

\section{Environnement de travail}

La disponibilité des infrastructures et du matériel de base nécessaires (coins pour les nouveau-nés, vidoir isolé pour les déchets, réfrigérateur) dans la salle d'accouchement fait défaut au niveau des sites visités avec un score moyen de 58 points. Le score moyen de disponibilité du matériel de soins du nouveau-né est faible (51 points) et ainsi que celle du matériel de prévention des infections (53 points). 
On observe aussi une faible disponibilité des médicaments et produits pour la GATPA avec un score moyen de 53 points. Les médicaments pour les SENN du nouveau-né sont plus disponibles mais le score moyen demeure faible (69 points). Certains produits comme le Misoprostol et la Vitamine $\mathrm{K}$ manquent et on relève des problèmes de conservation des produits tels que l'ocytocine qui de santé n'est pas conservée selon les normes dans $30 \%$ des structures.

\section{Qu'en est-il de la connaissance des procédures?}

Les données indiquent une connaissance des procédures de la GATPA parmi les prestataires (93,3\%). Concernant les SENN, la connaissance théorique des prestataires est très satisfaisante avec 8 prestataires sur 10 étant capables de citer les cinq éléments des soins de base du nouveau-né.

\section{Qu'en est-il de la pratique actuelle?}

Réalisée sur un échantillon de 170 cas, l'observation de cette pratique a montré que les trois éléments clés de la procédure GATPA sont bien suivis par les prestataires. Toutefois, il convient d'améliorer le suivi de certaines étapes non moins négligeables, notamment celle de la préparation dont le score reste faible.

\section{Score moyen de performance par type de prestataire pour la pratique de la GATPA}

Score pour la préparation $56 \%$

Score pour la délivrance $83 \%$

Score pour le massage utérin $66 \%$

Score pour les soins de base du nouveau-né $79 \%$

Score pour l'enregistrement des données

$73 \%$

Les résultats montrent également un suivi assez satisfaisant des 5 éléments clés de la procédure de prise en charge du nouveau-né après l'accouchement.

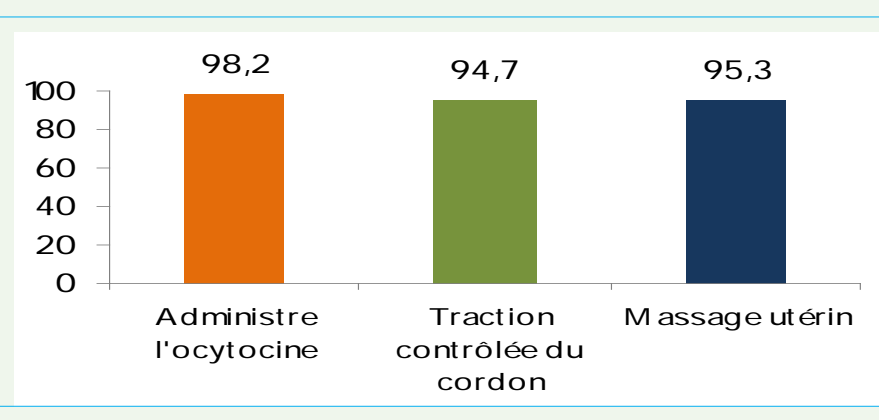

L'analyse des facteurs de performance révèle des dysfonctionnements au niveau de l'environnement de travail des prestataires. On note une insuffisance voire un manque de produits de la GATPA ainsi que ceux du nouveau-né au niveau des structures. Seul $29,6 \%$ des prestataires ont déclaré disposer de tout le matériel nécessaire pour la prise en charge du nouveau-né. Seulement $41 \%$ d'entre eux ont été supervisés par le niveau supérieur au cours des 3 derniers mois et parmi eux, $77 \%$ ont reçu un feedback immédiat sur leur performance.

\begin{tabular}{|l|r|}
\hline \multicolumn{2}{|l|}{ Pourcentage de prestataires ayant suivi les } \\
éléments clés de la prise en charge des SENN \\
\hline Maintient de la température & $87 \%$ \\
\hline Mise au sein précoce & $92 \%$ \\
\hline Soins du cordon & $81 \%$ \\
\hline Administration de la collyre & $92 \%$ \\
\hline Administration vitamine K1 & $69 \%$ \\
\hline
\end{tabular}

\section{Recommandations}

- Améliorer l'environnement de travail des prestataires pour consolider cette pratique au niveau des sites

- Renforcer la disponibilité des produits de la GATPA et des SENN et les conditions de conservation de ces intrants

- Promouvoir l'appui à la supervision afin de consolider les acquis

- Mettre l'accent sur la notification des données à travers un enregistrement systématique sur les supports de collecte pour faciliter la documentation.

Ce resume a été rendu possible grâce à l'appui apporté par le peuple américain 